\title{
Dog Bite Wound: A Dilemma into the Immediate Surgical Approach
}

\author{
Naveen Kumar ${ }^{1}$ \\ ${ }^{1}$ Department of Burns, Plastic, and Reconstructive Surgery, \\ Dr. Ram Manohar Lohia Hospital and Post Graduate Institute of \\ Medical Education and Research Hospital, New Delhi, India
}

Address for correspondence Naveen Kumar, MS, MCh Plastic Surgery, Department of Burns, Plastic, and Reconstructive Surgery, Dr. Ram Manohar Lohia Hospital and Post Graduate Institute of Medical Education and Research Hospital, New Delhi, 110001, India (e-mail: drnaveenvmmc@gmail.com).

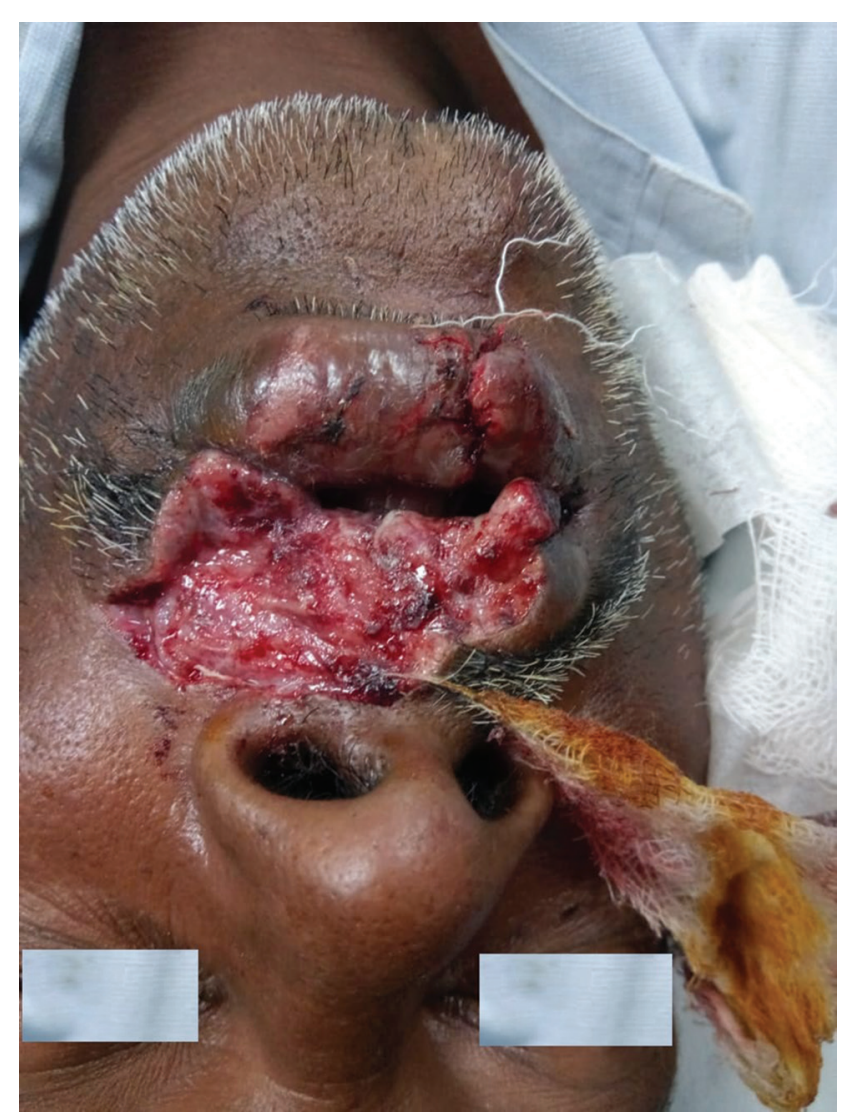

Fig. 1 Dog bite wound with significant tissue loss.

the body region. The study also noted a delay in presentation of more than 10 hours was associated with an increased risk of infection. ${ }^{1}$
DOI https://doi.org/

$10.1055 / \mathrm{s}-0040-1709914$ ISSN 0970-0358.
License terms

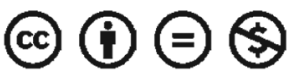




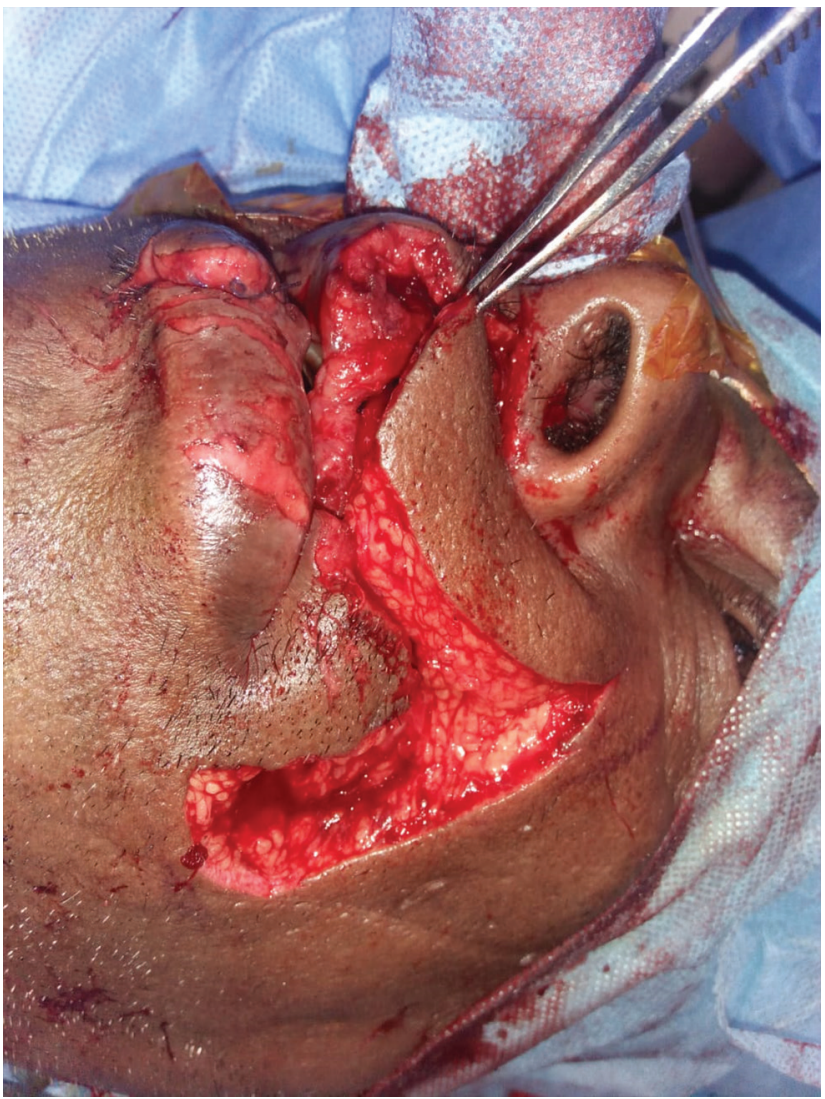

Fig. 2 Superiorly based nasolabial flap elevated.

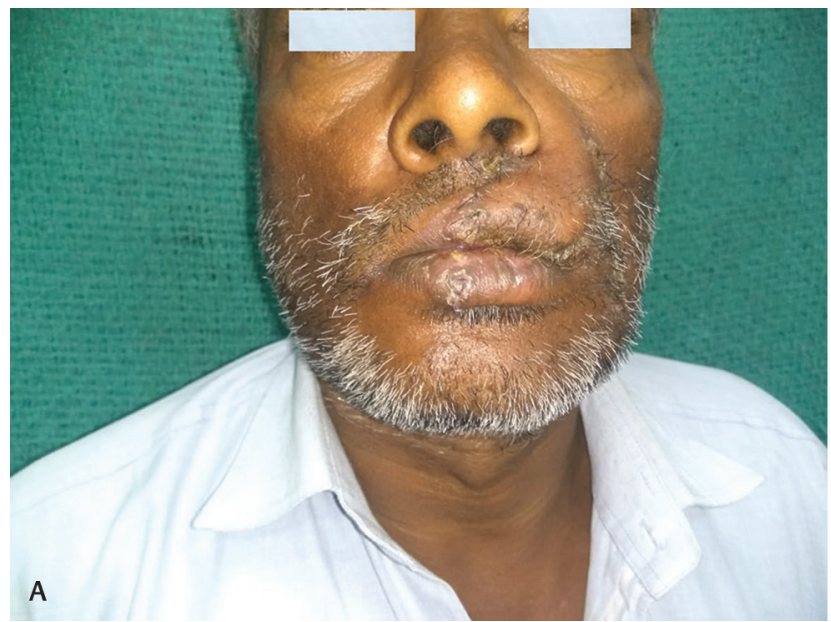

Fig. 4 Follow-up image after 6 weeks.

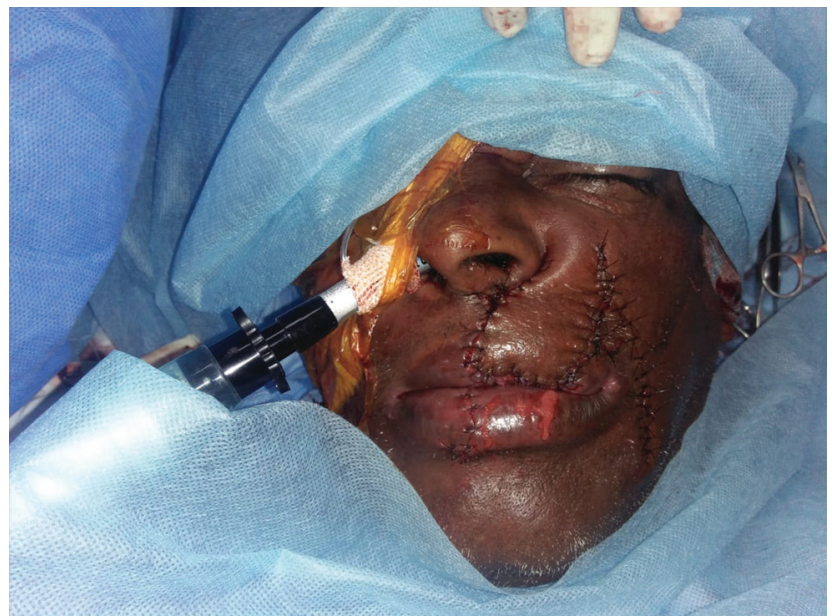

Fig. 3 Flap inset.

Facial dog bite in addition to being an infectious emergency also poses cosmetic and functional challenges. Therefore primary closure of the wound should be considered in the first place as it promotes the primary healing when compared with the open wound. ${ }^{2}$ The authors conclude that the primary closure of the dog bite wound, especially in a facial dog bite, should be considered particularly in an acute setting. For the rest of the wounds after thorough cleaning and debridement, especially hands or extremity wounds, decision should be taken depending on

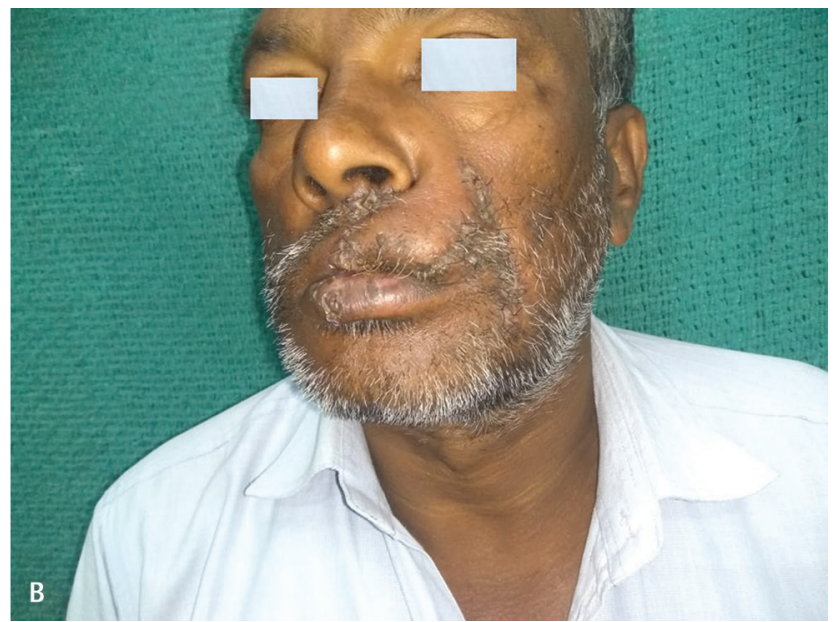


the time of presentation that whether the surgeon should opt for a single-stage primary closure or two-stage procedure, but an attempt toward the closure should be made in all cases.

In cases of crush injuries, complete avulsion or tissue loss where primary repair is not possible, reconstruction should be considered. In superficial low-risk wounds, especially in the region of the lip, primary repair may be considered. In deep wounds or avulsion injuries, if attempt to reattach the avulsed parts is unfortunately fails, a reconstruction using flaps should be considered. In cases of those wounds which are presented late, debridement of infected or necrosed tissues often lead to the substantial tissue loss which can be difficult to repair primarily but should be reconstructed using other available options.

\section{Conflict of Interest}

None declared.

\section{References}

1 Xiaowei Z, Wei L, Xiaowei H, et al. Comparison of primary and delayed wound closure of dog-bite wounds. Vet Comp Orthop Traumatol 2013;26(3):204-207

2 Rui-feng C, Li-song H, Ji-bo Z, Li-qiu W. Emergency treatment on facial laceration of dog bite wounds with immediate primary closure: a prospective randomized trial study. BMC Emerg Med 2013;13(Suppl 1):S2 